\title{
Muon spin rotation study of the magnetic penetration depth in the intercalated graphite superconductor $\mathbf{C a C}_{6}$
}

\author{
D. Di Castro, ${ }^{1}$ A. Kanigel,,${ }^{2,3}$ A. Maisuradze, ${ }^{4}$ A. Keren, ${ }^{2}$ P. Postorino, ${ }^{5}$ D. Rosenmann, ${ }^{6}$ U. Welp, ${ }^{7}$ G. Karapetrov, ${ }^{7}$ \\ H. Claus, ${ }^{7}$ D. G. Hinks, ${ }^{7}$ A. Amato, ${ }^{4}$ and J. C. Campuzano ${ }^{3,7}$ \\ ${ }^{1}$ CNR-SPIN and Dipartimento di Ingegneria Meccanica, Università di Roma Tor Vergata, Via del Politecnico 1, I-00133 Roma, Italy \\ ${ }^{2}$ Department of Physics, Technion, Haifa 32000, Israel \\ ${ }^{3}$ Department of Physics, University of Illinois at Chicago, Chicago, Illinois 60607, USA \\ ${ }^{4}$ Laboratory for Muon Spin Spectroscopy, Paul Scherrer Institut, CH-5232 Villigen PSI, Switzerland \\ ${ }^{5}$ Dipartimento di Fisica, Università di Roma La Sapienza, Piazzale Aldo Moro 2, I-00185 Roma, Italy \\ ${ }^{6}$ Center for Nanoscale Materials, Argonne National Laboratory, Argonne, Illinois 60439, USA \\ ${ }^{7}$ Materials Science Division, Argonne National Laboratory, Argonne, Illinois 60439, USA \\ (Received 1 March 2010; revised manuscript received 4 May 2010; published 26 July 2010)
}

\begin{abstract}
We report temperature- and magnetic field-dependent bulk muon spin rotation measurements in a $c$-axis-oriented superconductor $\mathrm{CaC}_{6}$ in the mixed state. Using both a simple second-moment analysis and the more precise analytical Ginzburg-Landau model, we obtained a field-independent in-plane magnetic penetration depth $\lambda_{a b}(0)=72(3) \mathrm{nm}$. The temperature dependencies of the normalized muon spin relaxation rate and of the normalized superfluid density result to be identical and both are well represented by the clean limit BCS model with $2 \Delta / \mathrm{k}_{B} T_{c}=3.6(1)$, suggesting that $\mathrm{CaC}_{6}$ is a fully gapped $\mathrm{BCS}$ superconductor in the clean limit regime.
\end{abstract}

DOI: 10.1103/PhysRevB.82.014530

PACS number(s): 74.70.Wz, 74.25.Ha, 76.75.+i

Recently the field of graphite intercalated compounds (GICs) has regained attention after the discovery of the superconducting GIC $\mathrm{CaC}_{6}$ with $T_{c} \sim 11.5 \mathrm{~K}$. $^{1,2}$ Soon after the discovery it was suggested ${ }^{3}$ that $\mathrm{CaC}_{6}$ is an unconventional superconductor and that the pairing interaction is of electronic origin. As time elapsed both calculations ${ }^{4}$ and experiments showed that $\mathrm{CaC}_{6}$ is a conventional BCS superconductor. In particular, experiments revealed the existence of a significant $\mathrm{Ca}$ isotope effect, ${ }^{5}$ the absence of gap nodes, ${ }^{6}$ a single gap $^{7}$ with BCS temperature dependence ${ }^{8}$ as well as the BCS temperature dependence of the London penetration depth. ${ }^{9}$ Moreover, calculations, ${ }^{4}$ supported by experimental evidence, ${ }^{6,7,10-13}$ showed the importance of a Ca derived interlayer band, crossing the Fermi energy, which has sufficiently strong coupling with both in-plane intercalant and out-of-plane graphite phonon modes to explain the $T_{c}$ of $\mathrm{CaC}_{6}$ within a standard electron-phonon coupling mechanism. On the other hand, the coupling between graphenederived electrons and high-frequency graphene-derived phonons was also demonstrated to be relevant. ${ }^{14}$

At present the mechanism of superconductivity in $\mathrm{CaC}_{6}$ and most of its properties have been investigated and clarified. Nevertheless a complete description of the superconducting state in a type-II superconductor requires a whole coherent set of parameters measured with high precision, such as the value of the coherence length $\xi$ and the magnetic penetration depth $\lambda$. These points are somehow still lacking. Indeed, two very different values of the in-plane penetration depth lambda $\lambda_{a b}(0)$ have been reported in the literature ${ }^{9,15}$ and the temperature-dependent superfluid density, $\rho(T)$, has been measured with surface-sensitive technique only. ${ }^{9}$ For the extremely air-sensitive superconductor $\mathrm{CaC}_{6}$, bulk measurement of the superconducting properties would be thus very important. We also notice that a new unambiguous bulk determination of $\rho(T)$ could also help in clarifying the nature of the $\mathrm{CaC}_{6}$ conduction regime, since both dirty-limit ${ }^{9,16,17}$ and clean-limit ${ }^{15,18}$ regimes have been reported so far. This system is also interesting from the point of view of $\mu \mathrm{SR}$ data analysis since it is a low $\kappa=\lambda / \xi$ superconductor with reduced in-field vortex phase.

The muon spin rotation $(\mu \mathrm{SR})$ technique is known to be one of the most indicated techniques to measure the bulk properties of type-II superconductors in the vortex state. In this paper we report a transverse-field muon spin rotation (TF- $\mu \mathrm{SR})$ experiment on high-quality $\mathrm{CaC}_{6} c$-axis-oriented samples, aimed to probe the vortex lattice and determine $\xi_{a b}(0), \lambda_{a b}(0)$, and the temperature dependence of the superfluid density $\rho(T) .{ }^{19}$ By using a peculiar method developed to collect reliable $\mu \mathrm{SR}$ data on very thin samples such as the $\mathrm{CaC}_{6}$ ones, we obtained $\lambda_{a b}(0)=72(3) \mathrm{nm}$ and $\xi_{a b}(0)$ $\simeq 38 \mathrm{~nm}$. The penetration depth results to be field independent, suggesting a fully gapped Fermi surface. The temperature dependence of the normalized superfluid density is very similar to that one of the normalized muon spin relaxation rate, as it is expected for a $\kappa \simeq 2$ superconductor at fields about $B_{c 2} / 2$. Both the temperature dependencies are well represented by the clean limit BCS model with $2 \Delta / \mathrm{k}_{B} T_{c}$ $=3.6(1)$, suggesting that $\mathrm{CaC}_{6}$ is a $\mathrm{BCS}$ superconductor in the clean-limit regime.

In TF- $\mu$ SR the sample is cooled down in a magnetic field, which here was parallel to the $c$ axis. The muons are implanted in the sample with their initial spin polarization almost perpendicular to the external magnetic field. Each muon precesses around to the local field. When the superconducting sample is in the mixed state, each muon probes a slightly different field. As a result, a dephasing process takes place and the average muon spin polarization decays. From the average polarization of the muon as a function of time, the parameters of the vortex lattice, $\lambda$ and $\xi$, can be extracted. $^{20,21}$ 

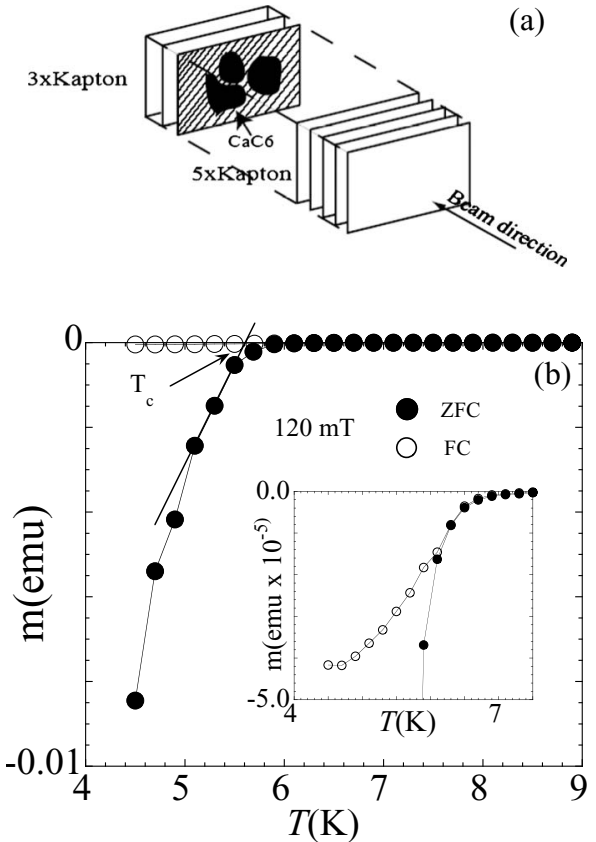

FIG. 1. (a) Illustration of the $\mathrm{CaC}_{6}$ sample and the Kapton stacks used to degrade the muon beam (see text). (b) Magnetic moment of $\mathrm{CaC}_{6}$ as a function of temperature measured at the same field used in TF- $\mu$ SR measurements (120 mT). The closed (open) symbols represent zero-field cooling (field cooling) measurements. Inset to panel (b): magnetic moment on enlarged scale to better show the ZFC data.

$\mathrm{CaC}_{6}$ samples were prepared using the alloy method as described by Emery et $a l .^{2}$ A stainless steel tube is loaded with lithium and calcium in the ratio 3:1. Natural graphite flakes are then added to the ampoule. The reaction takes place in an argon atmosphere for 10 days at $350{ }^{\circ} \mathrm{C}$. The flakes are typically of a size $2 \mathrm{~mm} \times 2 \mathrm{~mm}$ with a thickness of $50 \mu \mathrm{m}$. The low-field superconducting transition onset is at $T_{c}=11.6 \mathrm{~K}^{22}$ It was reported previously that these samples are very reactive and tend to degrade very fast. This is observed as an increase in the transition width and a decrease in the superconducting volume fraction. For that reason the samples were kept in an He atmosphere and overall were exposed to the room atmosphere for not more than a few minutes.

The experiments were done in the general purpose surface-muon (GPS) spectrometer at the Paul Scherrer Institute (PSI) Villigen, Switzerland. Three flakes were used, covering together an area of about $4 \mathrm{~mm} \times 4 \mathrm{~mm}$. The $\mathrm{CaC}_{6}$ samples are too thin to stop the muons. Given the muon stopping distance and the density of $\mathrm{CaC}_{6}$, a sample of a few hundred microns is needed for this purpose. To maximize the number of muons stopping in the samples, we used a method developed to measure very thin crystals. ${ }^{23}$ The samples were sandwiched between two stacks of Kapton foil rectangles. This assembly is illustrated in the panel (a) of Fig. 1. Each Kapton foil is $125 \mu \mathrm{m}$ thick. The role of the Kapton foils in front is to slow down the muons so that most of them will stop in the sample. The typical statistics of our $\mu \mathrm{SR}$ measurements was about 17 millions events.

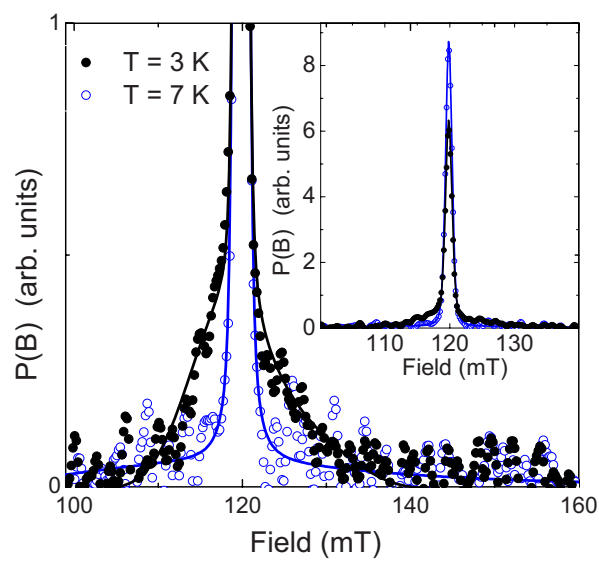

FIG. 2. (Color online) Magnetic field distribution $P(B)$ at an applied field of $120 \mathrm{mT}$ above $(7 \mathrm{~K})$ and below $(3 \mathrm{~K}) T_{c}$ obtained from the measured $\mu \mathrm{SR}$ time spectra by means of fast Fourier transform. The solid lines are fit by using Eq. (6). Inset: the same as in the main panel on larger scales.

Muons in Kapton are known to have a very high probability of forming muonium (muon-electron bound state). However, a muonium signal is not observed in our experiment. In addition, muons stopped by Kapton do not contribute substantially to the measured asymmetry since it is known that the asymmetry is strongly reduced by a thick Kapton layer. ${ }^{23}$ The Kapton foils in the back of the sample prevent slow muons that did not stop in the sample from stopping in the windows of the cryostat. In addition, we used a veto detector, that reduces the background signal by removing events from the data where the incoming muon missed the sample. Using the veto counter and Kapton combination we were able to get an asymmetry of about 0.1 , about half of that we estimated to be "good" signal coming from the $\mathrm{CaC}_{6}$ sample.

The $\mu \mathrm{SR}$ signal was recorded in the usual timedifferential way by counting positrons from decaying muons as a function of time. The time dependence of the positron rate is given by the expression ${ }^{24}$

$$
N(t)=N_{0} \frac{1}{\tau_{\mu}} e^{-t / \tau} \mu[1+a P(t)]+N_{b g},
$$

where $N_{0}$ is the normalization constant, $N_{b g}$ denotes the timeindependent background, $\tau_{\mu}=2.19703(4) \times 10^{-6} \mathrm{~s}$ is the muon lifetime, $a$ is the maximum decay asymmetry for the particular detector telescope and $P(t)$ is the polarization of the muon ensemble $P(t)=\int P(B) \cos \left(\gamma_{\mu} B t+\phi\right) d B$. Here $\gamma_{\mu}$ $=2 \pi \times 135.5342 \mathrm{MHz} / \mathrm{T}$ is the muon gyromagnetic ratio and $\phi$ is the angle between the initial muon polarization and the effective symmetry axis of a positron detector. $P(t)$ can be linked to the internal field distribution $P(B)$ by using the algorithm of Fourier transform. ${ }^{24}$

In Fig. 2 the magnetic field distribution $P(B)$ at an applied field of $120 \mathrm{mT}$ above $(7 \mathrm{~K})$ and below $(3 \mathrm{~K}) T_{c}$, obtained from the measured $\mu \mathrm{SR}$ time spectra by performing fast Fourier Transform, is shown. In the normal state, $P(B)$ is a sharp symmetric line centered at the position of the external magnetic field. Below $T_{c}$ the field-distribution results to be composed by two signals: one is represented by a sharp symmet- 
ric peak centered at the position of the external field and ascribed to different sources of background (cryostat window, Kapton foils), and the other is a broadened line, which signals the formation of a vortex lattice (VL) in the superconducting part of the sample. Indeed, VL makes the field in the sample highly inhomogeneous. This induces an increase in the muon spin relaxation rate $\sigma$, which is strictly related to the second moment of the field distribution. Moreover, the line shape of the superconducting $P(B)$ is asymmetric, as expected for a field distribution within a reasonably wellarranged VL. This could be questionable since recently it has been shown that the irreversibility line in $\mathrm{CaC}_{6}$ coincides with the $H_{c 2}(T)$ line ${ }^{25}$ which suggests that the pinning in this sample is very strong and that a large number of pinning sites are available. In the panel (b) of Fig. 1 the magnetic moment of $\mathrm{CaC}_{6}$, measured at the same field used in $\mathrm{TF}-\mu \mathrm{SR}$ measurements, is reported as a function of temperature in zero-field-cooling (ZFC) and field-cooling (FC) conditions. In the FC conditions the magnetic moment is negligible compared to the ZFC one, indicating a lack of field expulsion. The same was observed in the $\mu \mathrm{SR}$ experiment: we found almost no change in the average muon precession frequency when entering the superconducting phase. This is typically an effect of strong pinning, which confirms previous results. ${ }^{25}$ The regularity of the VL could be affected by the presence of pinning in the sample. The degree of the pinning-induced disorder of the flux line lattice (FLL) depends on many factors, as the strength and the nature of the pinning and the value of the external field. However, the asymmetric line shape of the measured field distribution in our samples indicates that, although a disorder of the FLL is present, it is not large enough to prevent a reliable analysis of the data.

As a first step, the $\mu \mathrm{SR}$ time spectra collected below $T_{c}$ were fitted by two Gaussian lines ${ }^{26}$

$$
\begin{aligned}
P(t)= & A_{b g} \exp \left(-\sigma_{b g}^{2} t^{2} / 2\right) \cos \left(\gamma_{\mu} B_{b g} t+\phi\right) \\
& +A \exp \left(-\sigma^{2} t^{2} / 2\right) \cos \left(\gamma_{\mu} B t+\phi\right),
\end{aligned}
$$

where $A_{b g}, \sigma_{b g}, B_{b g}$ and $A, \sigma, B$ are the asymmetry, the relaxation rate, and the mean field of the background signal and of the superconducting signal, respectively. The first one is assumed to be temperature and field independent. The second one is an approximation to the field distribution in the superconducting state of the sample. This distribution is both temperature and field dependent and it generates a Gaussian relaxation rate $\sigma(T)$ of the muon spin polarization, which is proportional to the second moment of the local-field distribution. The minimum field for which we could obtain a reliable value for $\sigma$ is $60 \mathrm{mT}$. Below this field the combination of a very fast relaxation and a relatively low frequency prevents a good fit.

In Fig. 3 we show the field dependence of $\sigma$ measured at $T \simeq 2 \mathrm{~K}$. The measurements were done in field-cooled conditions: for every field value the sample was warmed to a temperature above $T_{c}$ and cooled back to about $2 \mathrm{~K}$. As can

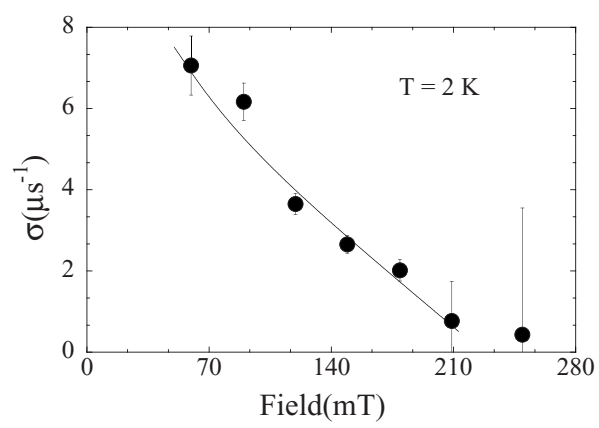

FIG. 3. Gaussian relaxation as a function of magnetic field at $T=2 \mathrm{~K}$. The solid line is a fit by Eq. (3).

be seen, there is an almost linear decrease in the relaxation rate with increasing field.

For a fully gapped BCS superconductor, as $\mathrm{CaC}_{6}$ is supposed to be, ${ }^{4,6,7,9}$ the magnetic penetration depth $\lambda$ should be field independent. ${ }^{27,28}$ In this case, by the analysis of the field dependence of $\sigma$ it is possible to obtain an estimate of the absolute value of $\lambda$, by using the approximation developed by Brandt [Eq. (13) in Ref. 29], which is considered a very good one for superconductors with $\kappa=\lambda / \xi \geq 5$ ( $\xi$ is the coherence length)

$$
\sigma \approx 0.172 \gamma_{\mu} \frac{\Phi_{0}}{2 \pi}\left(1-\frac{B}{B_{c 2}}\right)\left[1+1.21\left(1-\sqrt{\frac{B}{B_{c 2}}}\right)^{3}\right] \lambda_{a b}^{-2},
$$

where $\gamma_{\mu}=2 \pi \times 135.5342 \mathrm{MHz} / \mathrm{T}$ is the muon gyromagnetic ratio, $\Phi_{0}$ is the flux quantum, $B_{c 2}^{a b}$ the in-plane upper critical field, and $\lambda_{a b}$ the in-plane magnetic-field penetration depth. The model reproduces the data reasonably well (solid line in Fig. 3). This is thus consistent with a field independent $\lambda_{a b}$ and therefore with $\mathrm{CaC}_{6}$ being a fully gapped superconductor. The fit (done without considering the data point at $250 \mathrm{mT}$ which is too close or above $B_{c 2}$ and is compatible with the background value) gives $\lambda_{a b}$ $=77(3) \mathrm{nm}$ and $B_{c 2}=230(10) \mathrm{mT}$. The latter, within the Ginzburg-Landau picture, corresponds to $\xi_{a b} \simeq 38 \mathrm{~nm}$. Both the obtained parameters, $\xi_{a b}$ and $\lambda_{a b}$, are in good agreement with previous results $2,6,7,9,10,15,30$ and give $\kappa=\lambda_{a b} / \xi_{a b} \approx 2$. Although the $\kappa=\lambda / \xi=2$ is rather small the Eq. (3) is still valid in the fields $\sqrt{B / B_{c 2}} \geq 0.5$ (see Fig. 6 in Ref. 29), that is, in the range of our data.

These results have been obtained by using a Gaussian approximation for the field distribution $P(B)$. To obtain a more accurate value of $\lambda_{a b}$ in the following we will analyze the muon data by using the analytical Ginzburg-Landau (AGL) approximation, ${ }^{31,32}$ which is valid for a broad range of fields and of Ginzburg-Landau parameter $\kappa$.

The spatial-field distribution $\mathbf{B}(\mathbf{r})$ in the vortex lattice was calculated as follows:

$$
B(\mathbf{r})=\langle B\rangle \sum_{\mathbf{G}} \exp (-i \mathbf{G} \cdot \mathbf{r}) B_{\mathbf{G}}(\lambda, \xi,\langle B\rangle) .
$$

The $B_{\mathbf{G}}$ are the Fourier components $\mathrm{s}^{31,32}$ 


$$
B_{\mathbf{G}}=\frac{\Phi_{0}}{S} \frac{f_{\infty} K_{1}\left[\frac{\xi_{v}}{\lambda}\left(f_{\infty}^{2}+\lambda^{2} G^{2}\right)^{1 / 2}\right]}{\left(f_{\infty}^{2}+\lambda^{2} G^{2}\right)^{1 / 2} K_{1}\left(\frac{\xi_{v}}{\lambda} f_{\infty}\right)},
$$

with $f_{\infty}=1-b^{4}$ and

$$
\xi_{v}=\xi\left(\sqrt{2}-\frac{0.75}{\kappa}\right)\left(1+b^{4}\right)^{1 / 2}\left[1-2 b(1-b)^{2}\right]^{1 / 2} .
$$

Here, $\langle B\rangle$ is the average magnetic field inside the superconductor, $\xi$ the coherence length, $K_{1}(x)$ is the modified Bessel function, $\mathbf{r}$ the vector coordinate in a plane perpendicular to the applied field, $\mathbf{G}=4 \pi / \sqrt{3} a(m \sqrt{3} / 2, n+m / 2)$ the reciprocal lattice vector of the hexagonal FLL lattice, $a$ the intervortex distance, and $m$ and $n$ are integer numbers. From this spatialfield distribution the probability field distribution $P(B)$ was calculated.

The $\mu$ SR time spectra were fitted with the depolarization function $P(t)$ obtained from $P(B)$ as follows:

$$
P(t)=A e^{-1 / 2 \sigma_{g}^{2} t^{2}+i \phi} \int P(B) e^{i \gamma_{\mu} B t} d B+A_{b g} e^{-\sigma_{L} t+i\left(\gamma_{\mu} B_{a} t+\phi\right)},
$$

where the second term is the signal of the background with asymmetry $A_{B G}$ and relaxation $\sigma_{L}$. The parameter $\sigma_{g}$ describes the disorder of the FLL and was fixed proportional to $1 / \lambda^{2}$ such that $\sigma_{g}(0)=2.1 \mu \mathrm{s}^{-1}$ (note that this relaxation is much smaller than the superconducting relaxation, which are summed in quadrature). Such relation corresponds to the rigid (well pinned) FLL. ${ }^{21,33}$ We neglected additional nuclear relaxation since the concentration of $\mathrm{Ca}$ and $\mathrm{C}$ isotopes with magnetic nuclear moments is practically zero. The parameter $\xi$ was kept fixed during the fit at the values determined, at each temperature, from the corresponding $B_{c 2}(T)$ via the Ginzburg-Landau relation $\xi(T)=\sqrt{\left\{\Phi_{0} /\left[2 \pi B_{c 2}(T)\right]\right\}}$. The $B_{c 2}(T)$ values were obtained by linearly interpolating the following four experimental points: $B_{c 2}(2 K)=230 \mathrm{mT}$, given above by the fit of the field dependence of $\sigma$, and $B_{c 2}(5.6 K)=120 \mathrm{mT}, B_{c 2}(8.5 K)=60 \mathrm{mT}$, and $B_{c 2}(11.5 K)=0$ measured from magnetization experiments (only the measurement at $120 \mathrm{mT}$ is shown in Fig. 1). These experimental points agree well with the $B_{c 2}(T)$ curve reported by Cubitt et al. ${ }^{15}$ The AGL model represents very accurately the experimental data, as shown by the solid line in Fig. 2.

In Fig. 4, the values of $\lambda_{a b}$, obtained by fitting the $\mu \mathrm{SR}$ data with Eq. (6) at the lowest temperature and several fields, are shown. No field dependence of $\lambda_{a b}$ is detected within error bar, as expected for conventional fully gapped BCS superconductors and already suggested by the secondmoment analysis (Fig. 3). A weighted average of the data gives $\lambda_{a b}=72(3) \mathrm{nm}$ compatible, within the errors, with the value obtained by fitting the field dependence of the relaxation rate and in very good agreement with the value reported in Ref. 9. Recently Cubitt et al. ${ }^{15}$ reported a magnetic penetration depth $\lambda=50 \mathrm{~nm}$ using SANS on $\mathrm{CaC}_{6}$ compound. In their analysis they used the London model with Gaussian cut off (LGC). As it was shown previously by Yaouanc

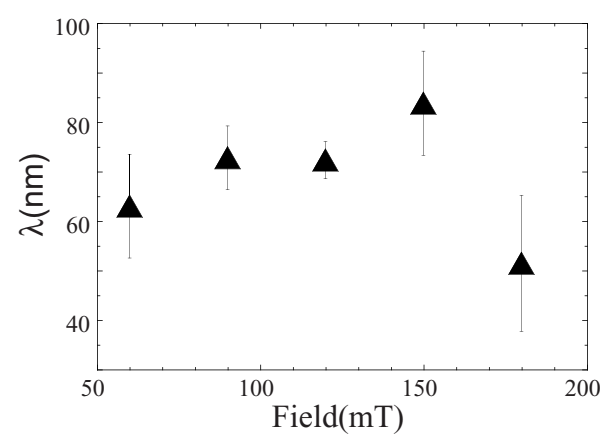

FIG. 4. Magnetic penetration depth as a function of magnetic field obtained from the fit to the $\mu$ SR time spectra at $2 \mathrm{~K}$.

et al. ${ }^{32}$ the form factors of the LGC model deviate substantially from the exact solution of the Ginzburg-Landau model in a very broad range of fields. Indeed, an analysis of our data by using the LGC model gives $\lambda_{a b}=52(4) \mathrm{nm}$, in good agreement with the results of Cubitt et al. ${ }^{15}$ The AGL model used here gives more reliable results ${ }^{32}$ and systematic deviations of the AGL model from the exact solution of the Ginzburg-Landau ${ }^{34}$ model are within the error bar.

The magnetic penetration depth is defined, in the London limit of low fields, as a measure of the superfluid density $\rho$ $\propto \lambda^{-2}$. However, for fields close to $B_{c 2}$ the mean magnitude of the Ginzburg-Landau order parameter $\psi(r)$ drops due to a substantial overlapping of the vortex cores. ${ }^{34}$ The spatial average of the superfluid density is thus well described by $y^{35,36}$

$$
\rho(T) \propto\left\langle|\psi(r)|^{2}\right\rangle \lambda^{-2}(T) \simeq\left[1-B / B_{c 2}(T)\right] \lambda^{-2}(T),
$$

where $\langle\cdots\rangle$ refers to the spatial average over the unit cell of the FLL. The temperature dependence of $\lambda$, needed to calculate $\rho(T)$ with Eq. (7), was obtained by fitting of Eq. (6) to the data collected at different temperatures in a field of 120 $\mathrm{mT}$. The $B_{c 2}(T)$ values were obtained as described above.

In Fig. 5, we show the normalized superfluid density $\rho(T) / \rho(0)$ as a function of the reduced temperature $T / T_{c}$ measured at $120 \mathrm{mT} . \rho(0)$ is obtained from the BCS fit to the temperature dependent $\rho(T)$ by using the equation ${ }^{37}$

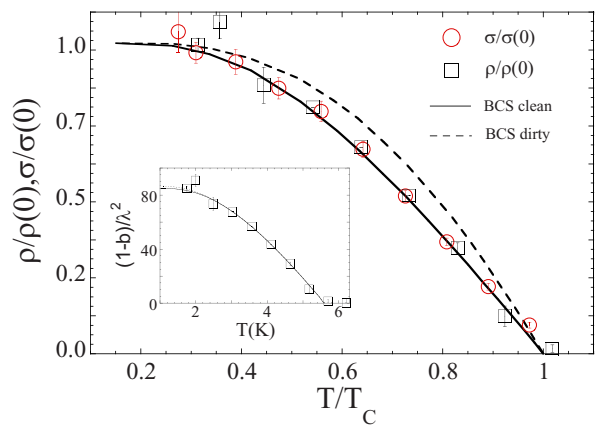

FIG. 5. (Color online) Normalized superfluid density $\rho(T) / \rho(0)$ and muon spin relaxation rate $\sigma(T) / \sigma(0)$ as a function of the reduced temperature $T / T_{c}$ measured at $120 \mathrm{mT}$. Full and dashed lines are BCS clean limit and dirty limit curves, respectively. Inset: temperature dependence of $\rho$ and BCS fits. 


$$
\rho(T)=\rho(0)\left[1-\frac{2}{k_{B} T} \int_{0}^{\infty} f[\varepsilon, T, \Delta(T)]\{1-f[\varepsilon, T, \Delta(T)]\} d \varepsilon\right],
$$

where $f(\varepsilon, T, \Delta(T))=\left[1+\exp \left[\sqrt{\varepsilon^{2}+\Delta^{2}(T)} /\left(k_{B} T_{C}\right)\right]\right]^{-1}$ is the Fermi distribution and $\Delta(T)=\Delta(0) \delta\left(T / T_{c}\right)$ with $\delta\left(T / T_{c}\right)$ given by the conventional BCS temperature dependence of the gap. ${ }^{38}$ Indeed, although data at temperatures lower than $1.75 \mathrm{~K}$ would be important to clearly assess the occurrence of a saturation, however the absence of a field dependence of $\lambda$ points to nodeless gap in the $a b$ plane of $\mathrm{CaC}_{6}$, which is consistent with previous theoretical end experimental studies. ${ }^{4,6,7,9}$ Therefore it is correct to fit the experimental data using Eq. (8). In order to reduce the number of fitting parameters, the fit has been performed by keeping $T_{c}$ fixed at the value 5.6(1) $\mathrm{K}$ obtained from magnetization data at the same field of $120 \mathrm{mT}$ (see Fig. 1). As shown in the inset to Fig. 5, the fitting curve (solid line) represents well the experimental data, yielding $\rho(0)=85.1(3) \mu \mathrm{m}^{-2}$ and $\Delta(0)$ $=0.868(5) \mathrm{meV}$, which gives $2 \Delta(0) / \mathrm{k}_{B} T_{c}=3.6(1)$. This value is compatible with the one expected for a weak coupling BCS superconductor and is in good agreement with previous results. ${ }^{6,7,9}$ A fit with the dirty limit BCS temperature dependence ${ }^{37}$ is also acceptable (see inset to Fig. 5) although with larger $\chi^{2}$ and larger errors of the fitting parameters $\left[\rho(0)=87(2) \mu \mathrm{m}^{-2}, \Delta(0)=0.66(2) \mathrm{meV}\right]$. With these parameters we would obtain $2 \Delta / \mathrm{k}_{B} T_{c}=2.74(1)$. This quantity is substantially different from the previously reported ones $^{6,7,9}$ and it is well below the BCS weak-coupling ratio. The normalized superfluid density, shown in the main panel of Fig. 5, clearly follows the clean limit curve and is not compatible with the dirty limit one, suggesting that bulk $\mathrm{CaC}_{6}$ is a $\mathrm{BCS}$ superconductor in the clean limit regime, in agreement with Mialitsin et al. ${ }^{18}$ and Cubitt et al. ${ }^{15}$

In the main panel of Fig. 5 the temperature dependence of the normalized relaxation rate $\sigma(T)$, obtained by the twoGaussian model [Eq. (2)], is also reported. These data closely resemble the $\rho(T)$ curve. Indeed, as follows from the Fig. 6 of Ref. 29 the quantity $\sigma \kappa^{2} / B_{c 2}(1-b) \propto \sigma /\left[(1-b) / \lambda^{2}\right]$ is practically independent of the reduced field $b=\langle B\rangle / B_{c 2}$ for $\kappa \simeq 2$ and $\sqrt{b}>0.25$. On the other hand, $\sigma /\left[(1-b) / \lambda^{2}\right]$ is proportional to $\sigma / \rho$. Therefore, the present case with $\kappa \simeq 2$ is exceptional, when a simple second moment analysis gives a quite precise result for the temperature dependence of the superfluid density in a broad range of fields.

To summarize, we performed TF- $\mu \mathrm{SR}$ measurements on the intercalated graphite superconductor $\mathrm{CaC}_{6}$ in the vortexlattice state. Our experimental method included the use of Kapton tape to gather good data from very thin samples, $50 \mu \mathrm{m}$ thick. Despite of strong pinning, we detected an asymmetric field distribution, typically observed in superconducting single crystals in the vortex state. The data were analyzed with two different models. Using a simple twoGaussian model, the relaxation rate $\sigma$ was obtained. Its field dependence suggested a field independent $\lambda_{a b}(0)$ $=77(3) \mathrm{nm}$ and a coherence length $\xi_{a b}(0) \simeq 38 \mathrm{~nm}$. The more precise analytical Ginzburg-Landau model yielded $\lambda_{a b}(0)=72(3) \mathrm{nm}$, a value remarkably close to that one obtained by the second-moment analysis and in good agreement with previous microwave measurements. ${ }^{9}$ Also this analysis reveals that $\lambda_{a b}(0)$ does not depend on magnetic field, suggesting a fully gapped superconductivity in $\mathrm{CaC}_{6}$, in agreement with previous reports. ${ }^{6,7,9}$ The temperature dependence of the superfluid density was analyzed by both the clean and the dirty limit BCS models. The experimental data are well fitted by the clean limit one, giving a gap-to- $T_{c}$ ratio $2 \Delta(0) / \mathrm{k}_{B} T_{c}=3.6(1)$, in agreement with previous results obtained by surface sensitive techniques. ${ }^{7,9}$ The temperature dependence of the normalized superfluid density results to be identical to the temperature dependence of the normalized relaxation rate, as it should be for a $\kappa \simeq 2$ superconductor at fields $B>0.1 B_{c 2}$. Both are well represented by the clean limit $\mathrm{BCS}$ curve, suggesting that $\mathrm{CaC}_{6}$ is an $s$-wave BCS superconductor in the clean limit regime.

This work was partly performed at the Swiss Muon Source $(\mathrm{S} \mu \mathrm{S})$, Paul Scherrer Institute (PSI, Switzerland). The authors are grateful to R. Khasanov for useful discussions. This work was partly supported by the EU Project $\mathrm{CoMePhS}$, by the Israel Science Foundation and by the European Commission under the sixth Framework Programme through the Key Action: Strengthening the European Research Area, Research Infrastructures; Contract No. RII3-CT2003-505925. The work at Argonne National Laboratory was supported by UChicago Argonne, LLC, Operator of Argonne National Laboratory (Argonne). Argonne, a U.S. Department of Energy, Office of Science laboratory is operated under Contract No. DE-AC02-06CH11357.
${ }^{1}$ T. E. Weller, M. Ellerby, S. S. Saxena, R. P. Smith, and N. T. Skipper, Nat. Phys. 1, 39 (2005).

${ }^{2}$ N. Emery, C. Hérold, M. d'Astuto, V. Garcia, Ch. Bellin, J. F. Marêché, P. Lagrange, and G. Loupias, Phys. Rev. Lett. 95, 087003 (2005).

${ }^{3}$ G. Csányi, P. B. Littlewood, A. H. Nevidomskyy, C. J. Pickard, and B. D. Simons, Nat. Phys. 1, 42 (2005).

${ }^{4}$ I. I. Mazin, Phys. Rev. Lett. 95, 227001 (2005); M. Calandra and F. Mauri, ibid. 95, 237002 (2005).

${ }^{5}$ D. G. Hinks, D. Rosenmann, H. Claus, M. S. Bailey, and J. D.
Jorgensen, Phys. Rev. B 75, 014509 (2007).

${ }^{6}$ J. S. Kim, R. K. Kremer, L. Boeri, and F. S. Razavi, Phys. Rev. Lett. 96, 217002 (2006).

${ }^{7}$ N. Bergeal, V. Dubost, Y. Noat, W. Sacks, D. Roditchev, N. Emery, C. Hérold, J-F. Marêché, P. Lagrange, and G. Loupias, Phys. Rev. Lett. 97, 077003 (2006).

${ }^{8}$ R. S. Gonnelli, D. Daghero, D. Delaude, M. Tortello, G. A. Ummarino, V. A. Stepanov, J. S. Kim, R. K. Kremer, A. Sanna, G. Profeta, and S. Massidda, Phys. Rev. Lett. 100, 207004 (2008).

${ }^{9}$ G. Lamura, M. Aurino, G. Cifariello, E. Di Gennaro, A. Andre- 
one, N. Emery, C. Hérold, J.-F. Marêché, and P. Lagrange, Phys. Rev. Lett. 96, 107008 (2006).

${ }^{10}$ R. Xie, D. Rosenmann, A. Rydh, H. Claus, G. Karapetrov, W. K. Kwok, and U. Welp, Physica C 439, 43 (2006).

${ }^{11}$ A. Sanna, G. Profeta, A. Floris, A. Marini, E. K. U. Gross, and S. Massidda Phys. Rev. B 75, 020511(R) (2007).

${ }^{12}$ K. Sugawara, T. Sato, and T. Takahashi, Nat. Phys. 5, 40 (2009).

${ }^{13}$ H. Okazaki, R. Yoshida, K. Iwai, K. Noami, T. Muro, T. Nakamura, T. Wakita, Y. Muraoka, M. Hirai, F. Tomioka, Y. Takano, A. Takenaka, M. Toyoda, T. Oguchi, and T. Yokoya, Phys. Rev. B 80, 035420 (2009).

${ }^{14}$ T. Valla, J. Camacho, Z.-H. Pan, A. V. Fedorov, A. C. Walters, C. A. Howard, and M. Ellerby, Phys. Rev. Lett. 102, 107007 (2009).

${ }^{15}$ R. Cubitt, J. S. White, M. Laver, M. R. Eskildsen, C. D. Dewhurst, D. McK. Paul, A. J. Crichton, M. Ellerby, C. Howard, Z. Kurban, and F. Norris, Phys. Rev. B 75, 140516(R) (2007).

${ }^{16}$ U. Nagel, D. Hüvonen, E. Joon, J. S. Kim, R. K. Kremer, and T. Rõõm, Phys. Rev. B 78, 041404(R) (2008).

${ }^{17}$ F. Murányi, G. Urbanik, V. Kataev, and B. Büchner, Phys. Rev. B 77, 024507 (2008).

${ }^{18}$ A. Mialitsin, J. S. Kim, R. K. Kremer, and G. Blumberg, Phys. Rev. B 79, 064503 (2009).

${ }^{19}$ The measurements were performed into two runs. The second run was needed in order to have additional points at 1.75 and 5.7 $\mathrm{K}$ in the temperature dependence of the superfluid density. Also the point at $2 \mathrm{~K}$ was measured a second time and the point shown in this manuscript comes from the average of the parameters obtained by the fit of the two runs.

${ }^{20}$ J. E. Sonier, J. H. Brewer, and R. F. Kiefl, Rev. Mod. Phys. 72, 769 (2000)

${ }^{21}$ A. Maisuradze, R. Khasanov, A. Shengelaya, and H. Keller, J. Phys.: Condens. Matter 21, 075701 (2009).

${ }^{22}$ C. Kurter, L. Ozyuzer, D. Mazur, J. F. Zasadzinski, D. Rosenmann, H. Claus, D. G. Hinks, and K. E. Gray, Phys. Rev. B 76, 220502(R) (2007).
${ }^{23}$ G. Allodi, R. De Renzi, S. Serventi, D. Di Castro, and R. Khasanov (unpublished).

${ }^{24}$ A. Schenck, Muon Spin Rotation: Principles and Applications in Solid State Physics (Adam Hilger, Bristol, 1986); S. F. J. Cox, J. Phys. C 20, 3187 (1987); J. H. Brewer, Encyclopedia of Applied Physics (VCH, New York, 1995), Vol. 11, p. 23.

${ }^{25}$ Ulrich Welp, Daniel Rosenmann, Ruobing Xie, David Hinks, Helmut Claus, Goran Karapetrov, John Schlueter, Wai-Kwong Kwok, and Lisa Paulius, http://meetings.aps.org/link/ BAPS.2007.MAR.B9.7

${ }^{26}$ R. Khasanov, D. G. Eshchenko, D. Di Castro, A. Shengelaya, F. La Mattina, A. Maisuradze, C. Baines, H. Luetkens, J. Karpinski, S. M. Kazakov, and H. Keller, Phys. Rev. B 72, 104504 (2005).

${ }^{27}$ R. Kadono, J. Phys.: Condens. Matter 16, S4421 (2004).

${ }^{28}$ I. L. Landau and H. Keller, Physica C 466, 131 (2007).

${ }^{29}$ E. H. Brandt, Phys. Rev. B 68, 054506 (2003).

${ }^{30}$ E. Jobiliong, H. D. Zhou, J. A. Janik, Y.-J. Jo, L. Balicas, J. S. Brooks, and C. R. Wiebe, Phys. Rev. B 76, 052511 (2007).

${ }^{31}$ Z. Hao, J. R. Clem, M. W. McElfresh, L. Civale, A. P. Malozemoff, and F. Holtzberg, Phys. Rev. B 43, 2844 (1991).

${ }^{32}$ A. Yaouanc, P. Dalmas de Réotier, and E. H. Brandt, Phys. Rev. B 55, 11107 (1997).

${ }^{33}$ T. M. Riseman, J. H. Brewer, K. H. Chow, W. N. Hardy, R. F. Kiefl, S. R. Kreitzman, R. Liang, W. A. MacFarlane, P. Mendels, G. D. Morris, J. Rammer, J. W. Schneider, C. Niedermayer, and S. L. Lee, Phys. Rev. B 52, 10569 (1995).

${ }^{34}$ E. H. Brandt, Phys. Rev. Lett. 78, 2208 (1997).

${ }^{35}$ A. Maisuradze, M. Nicklas, R. Gumeniuk, C. Baines, W. Schnelle, H. Rosner, A. Leithe-Jasper, Yu. Grin, and R. Khasanov, Phys. Rev. Lett. 103, 147002 (2009).

${ }^{36}$ P. Lipavský, J. Koláček, K. Morawetz, and E. H. Brandt, Phys. Rev. B 65, 144511 (2002).

${ }^{37}$ M. Tinkham, Introduction to Superconductivity (Krieger, Malabar, Florida, 1975).

${ }^{38}$ B. Mühlschlegel, Z. Phys. 155, 313 (1959). 